\title{
Study of sodium metal plasmon using electron energy loss spectroscopy
}

Shize Yang ${ }^{1}$ and Peter Crozier ${ }^{2}$

${ }^{1}$ Arizona State University, United States, ${ }^{2}$ Arizona State University, Tempe, Arizona, United States

Plasmon excitation in metal nanostructures have been widely studied for potential plasmonic device applications. Surface plasmon effects that can be tuned by changing the material, size and shape attracted great research interest [1]. Electron energy-loss spectroscopy (EELS) is a powerful tool to study optical properties of materials with high spatial resolution [1,2]. Experimental and theoretical work has been carried out on the plasmon effect of metal clusters [3]. The plasmon effect of sodium clusters in the range of Na20 to Na300 has been investigated theoretically and experiments have been done for Na20 and N92 [4,5]. The electron beam can reduce $\mathrm{NaCl}$ and form $\mathrm{Na}$ clusters inside the transmission electron microscopes (TEM). By controlling the electron beam current, electron beam position and interaction time, different sizes of $\mathrm{Na}$ particles can be produced in-situ. These clusters and particles can then be directly imaged in the TEM and the plasmon effect can be directly probed through EELS.

In our experiment, we dispersed commercial $\mathrm{NaCl}$ powder into IPA solvent and dropped a few droplets onto a TEM grid. The sample was then baked at $1200 \mathrm{C}$ for 8 hours before putting into a Nion microscope for EELS study. We used a $60 \mathrm{kV}$ probe with convergence semi-angle of $19 \mathrm{mrad}$, beam current of around $2 \mathrm{pA}, 1 \mathrm{~mm}$ EELS aperture and zero loss peak at around $9 \mathrm{meV}$ at FHWM. We first collected EELS spectrum with two different configurations: one is in aloof mode with an impact parameter of around 10 $\mathrm{nm}$ and the other is in transmission mode at the very edge of the $\mathrm{NaCl}$ cube particle. The two typical spectra are shown in Figure 1.

As shown in Figure 1. In the aloof mode, two peaks at around $3.3 \mathrm{eV}$ and $8 \mathrm{eV}$ are clearly observed. The $8 \mathrm{eV}$ peak is assigned to the exciton absorption of $\mathrm{NaCl}$ crystal and the peak at $3.3 \mathrm{eV}$ is attributed to the surface plasmon of small sodium particles formed during sample preparation. In the transmission mode, several additional peaks arise. The peak near $6 \mathrm{eV}$ is assigned to the volume plasmon of sodium particles. The peaks around $1.6 \mathrm{eV}$ and $2.5 \mathrm{eV}$ were found to be highly dynamic as the sample underwent damage due to electron beam. In-situ EELS results are further recorded, and the evolution of these peaks will be correlated with the formation of $\mathrm{Na}$ clusters and agglomeration of sodium clusters to sodium particles.

Acknowledgement:

We acknowledge the use of facilities within the Eyring Materials Center at Arizona State University supported in part by NNCI-ECCS-1542160. 

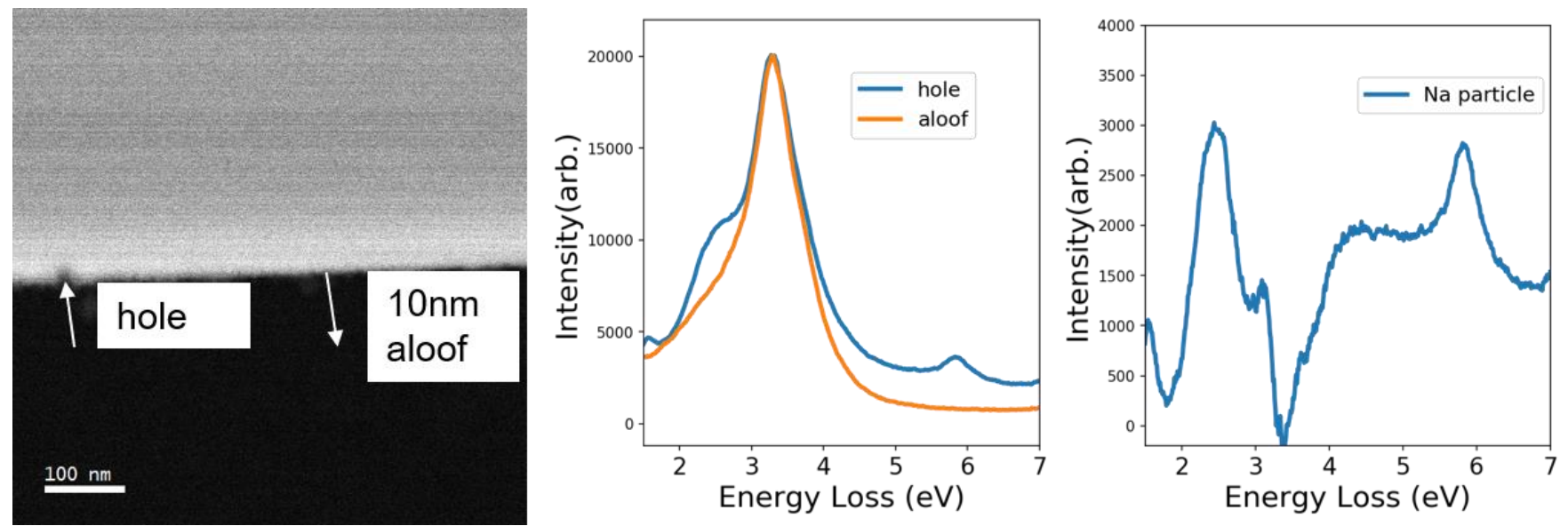

Figure 1. Figure 1. STEM ADF images showing the position of electron beams for measuring the electron energy loss spectroscopy and two spectra showing different features from two locations, the EELS spectrum of sodium particle was calculated by subtracting the aloof signal from the hole position signal, further subtraction taking consideration of impact factor will be further developed to better calculate the energy loss due to sodium particles.

\section{References}

[1] Campos, Alfredo, Nicolas Troc, Emmanuel Cottancin, Michel Pellarin, Hans-Christian Weissker, Jean Lermé, Mathieu Kociak, and Matthias Hillenkamp. "Plasmonic quantum size effects in silver nanoparticles are dominated by interfaces and local environments." Nature Physics 15, no. 3 (2019): 275280.

[2] De Abajo, FJ García. "Optical excitations in electron microscopy." Reviews of modern physics 82.1 (2010): 209.

[3] Brack, Matthias. "The physics of simple metal clusters: self-consistent jellium model and semiclassical approaches." Reviews of modern physics 65, no. 3 (1993): 677.

[4] Matsko, N. L. Formation of normal surface plasmon modes in small sodium nanoparticles. Physical Chemistry Chemical Physics, 22, no. 23 (2020): 13285-13291.

[5] Xia, Chunlei, Chunrong Yin, and Vitaly V. Kresin. "Photoabsorption by volume plasmons in metal nanoclusters." Physical review letters 102, no. 15 (2009): 156802. 